\title{
Deep Eutectic Solvents on the Surface of Face Centered Cubic Metals
}

\author{
Mert Atilhan, ${ }^{\mathrm{a}^{*}}$ and Santiago Aparicio ${ }^{* \mathrm{~b}}$ \\ ${ }^{a}$ Department of Chemical Engineering, Qatar University, P.O. Box 2713, Doha, Qatar \\ ${ }^{\mathrm{b}}$ Department of Chemistry, University of Burgos, 09001 Burgos, Spain \\ *Corresponding authors: $\underline{\text { sapar@ubu.es , +34 } 947258062 \text { (S.A.) and mert.atilhan@qu.edu.qa }}$ \\ ,_974 44034142 (M. A.)
}

\section{Supporting Information}


Table S1. Lattice parameters for FCC unit cells obtained from GGA-PBE / DNP theoretical calculations and the corresponding experimental data.

\begin{tabular}{|c|c|c|}
\hline & GGA-PBE / DNP & experimental $^{a}$ \\
\hline metal & $a=b=c$ & $a=b=c$ \\
\hline $\mathrm{Ag}$ & 4.0857 & 4.062 \\
\hline $\mathrm{Al}$ & 4.0495 & 4.019 \\
\hline $\mathrm{Pt}$ & 3.9239 & 3.913 \\
\hline
\end{tabular}

${ }^{a}$ Ref.

Table S2. Metal surfaces (100) used for molecular dynamics simulations. $N$ stands for the number of metal atoms in each system, and $L$ and $d$ the dimensions of the surfaces as defined in Figure S1.

\begin{tabular}{cccc}
\hline metal & $L / \AA$ & $d / \AA$ & $N$ \\
\hline $\mathrm{Ag}$ & 53.11 & 8.17 & 1823 \\
$\mathrm{Al}$ & 52.64 & 8.10 & 1823 \\
$\mathrm{Pt}$ & 54.94 & 7.85 & 2103 \\
\hline
\end{tabular}

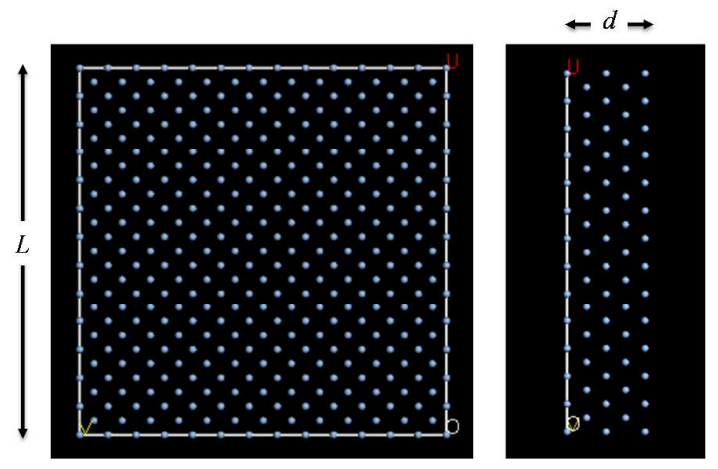

Figure S1. Ag (100) surface, top (left) and side (right) views, used for molecular dynamics simulations. Analogous surfaces are considered for the other metals with dimensions reported in Table S2.

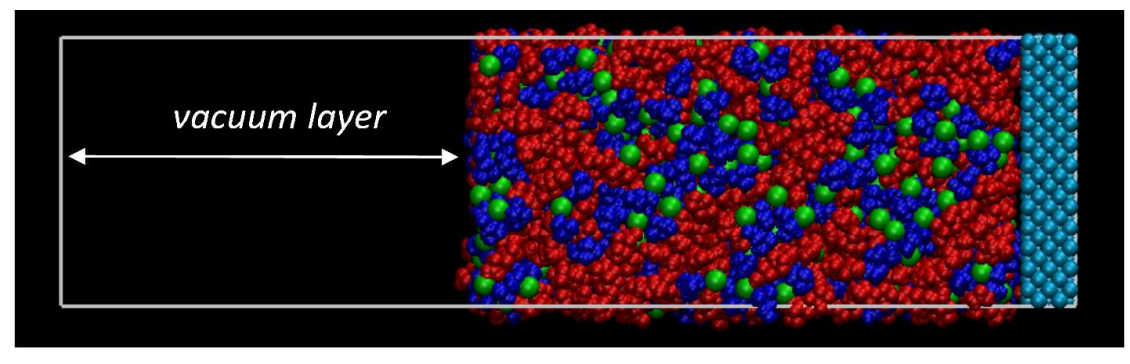

Figure S2. Ag (100) + DES-LEV used as initial simulation box for molecular dynamics simulations. Analogous systems were built for the eight metal surfaces. Color code: (blue) $[\mathrm{Ch}]^{+}$, (green) $[\mathrm{Cl}]^{-}$, (red) LEV, and (cyan) Ag. White lines shows periodic boundary conditions. A vacuum layer is placed above the DES-LEV liquid layer to avoid interactions with neighbour metal layers. 

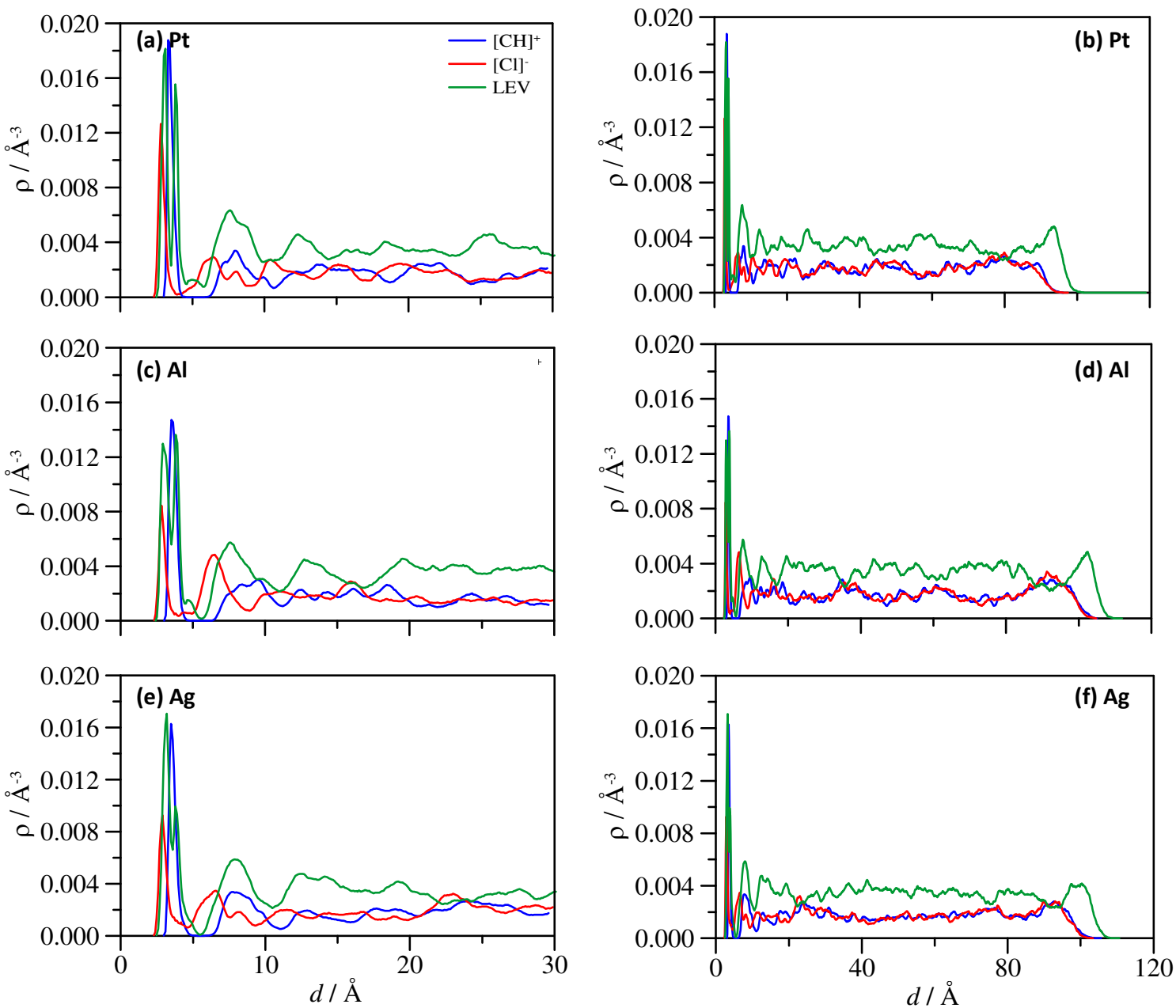

Figure S3. Number density profiles, $\rho$, for the center of mass of ions and molecules in DES-LEV as a function of the distance, $d$, to the corresponding metal surface. Plots in the left column shows results for $d<30$ and on the right column for the whole range including vacuum interfaces (right side of the plots), this is done to improve visibility of the data.

\section{REFERENCES}

(1) Haas, P.; Tran, F.; Blaha, P. Calculating of the Lattice Constant of Solids with Semilocal Functionals. Phys. Rev. B 2009, 79, 085104. 